\title{
Effects of Collagen Crosslink Augmentation on Mechanism of Compressive Load Sharing in Intervertebral Discs
}

\author{
Thomas P. Hedman' ${ }^{1}$ Weng-Pin Chen ${ }^{2}$ Leou-Chyr Lin ${ }^{3}$ Hsiu-Jen Lin ${ }^{2}$. \\ Shih-Youeng Chuang ${ }^{3,4}$
}

Received: 14 December 2015/Accepted: 2 June 2016/Published online: 19 January 2017

(C) The Author(s) 2018, corrected publication September 2018

\begin{abstract}
Exogenous crosslinking has been shown to have potential for treating disc degeneration and back pain due to its ability to increase the strength and toughness of the annulus fibrosus, increase intervertebral joint stability, decrease intradiscal pressure, and increase fluid flow through the disc. Some results imply that crosslink augmentation may also lead to changes in the compressive load sharing properties of the disc. The objective of the present study was to evaluate directional stress distribution changes of the disc following genipin crosslinking treatment. Bovine lumbar motion segments were randomly divided into control and crosslinked groups. Annular strains were determined from simultaneous deformation measurements at various time points during compressive creep testing. Four stress components of the annulus were then calculated according to the previously measured modulus data. Immediately after the application of a 750-N compressive load, mean axial and radial compressive stresses in the crosslinked group were twofold higher than control means. Conversely, mean lamellae-aligned and
\end{abstract}

The original version of this article was revised due to a retrospective Open Access order.

Shih-Youeng Chuang

shihyouc@hotmail.com

1 Department of Biomedical Engineering, University of Kentucky, Lexington, KY, USA

2 Department of Mechanical Engineering, National Taipei University of Technology, Taipei, Taiwan, ROC

3 Department of Orthopaedic Surgery, Tri-Service General Hospital, National Defense Medical Center, Taipei, Taiwan, ROC

4 Department of Orthopaedic Surgery, Kang-Ning General Hospital, No.26, Ln. 420, Sec. 5, Chenggong Rd., Neihu Dist., Taipei, Taiwan, ROC circumferential tensile stresses of the crosslinked discs were 8and threefold lower, respectively, compared to control means. After 1-h creep loading, the two compressive mean stresses in both the control and genipin-crosslinked specimens increased approximately threefold from their initial 750-N-loaded values. The two tensile mean stresses in the crosslinked group remained lower than the respective levels of the control means after creep loading. A greater proportion of annular compressive load support under compressive creep loading, with a commensurate decrease in both tensile stresses and strains, was seen in the discs following exogenous crosslink augmentation.

Keywords Intervertebral disc - Crosslink augmentation Compressive creep loading · Load sharing · Stress distribution

\section{Introduction}

Extracellular matrix (ECM) modification, including agerelated tissue changes, can effect stress distributions and mechanisms of load sharing in intervertebral joints [1-3]. Intervertebral discs are considered to work synergistically with the posterior elements and ligamentous structures for providing passive restraint capabilities of the spinal motion segment [4]. Modification of the disc ECM could potentially result in changes to the passive load support mechanism of the spinal motion segment. In addition, it is well known that the cells in the disc respond to mechanical stimulation through mechanoreceptors that modulate cellmatrix and cell-cell interactions. Consequently, matrix modification could affect the mechanical stimulation of cells and the response of cells to physiological loading, which can lead to a further modification of the ECM [5-7]. Studies have demonstrated that the cell growth rate and 
ECM production may be affected by mechanical stimulation [8-10]. In animal in vivo studies, it was shown that static compressive loading in the disc (immobilization) may cause harmful responses such as disorganization of the annulus fibrosus, an increase in apoptosis, associated losses of cellularity, and changes in the regulation of either proteoglycan, type I, or type II collagen [11-14].

One of the primary roles of the spinal motion segment in humans is to withstand compressive loads. The nucleus pulposus (NP) is constrained radially by the annulus fibrosus (AF) and caudally and cranially by cartilaginous endplates. With an increase in compressive loading, there is an interaction between the NP and the AF as the NP bulges outwards and is constrained by the AF. The NP acts hydrostatically with a linear proportional increase in intradiscal pressure (IDP) corresponding to an increase in the magnitude of compressive load [15]. The increased pressurization of the NP with corresponding axial deformation and radial disc bulge produces multidirectional stresses in the annular lamellae. Assuming quasi-static equilibrium in the axial plane, the applied compressive load on the disc will be directly resisted by the AF axial compressive force and a force produced by the hydrostatic pressure in the NP. Also associated with compressive loading conditions, the disc internal pressure exerted by the NP tends to stretch the annular lamellae outwards, but the tensile properties of the lamellar collagen resist this stretch. Assuming quasi-static equilibrium in the transverse plane, the transverse force due to NP hydrostatic pressure is a function of the radial compressive properties of the AF and of the circumferential and lamellae-aligned tensile forces of the bulging AF.

In this study, the effect of exogenous crosslinking using genipin on the load sharing properties of intervertebral discs was investigated. Collagen crosslinks are known to have an important role in contributing to the mechanical strength of load-supporting tissues $[16,17]$. Human discs have a higher concentration of mature crosslinks (enzymatically derived) than other tissues, which may be an indication of their demanding mechanical requirements $[17,18]$. With disc aging and degeneration, total collagen crosslinking increases; the quantity of mature crosslinks is decreased or unchanged, while the quantity of age-related crosslinks (non-enzymatically initiated by glycation, i.e., pentosidine) is increased [18, 19]. These data show that a natural, endogenous crosslink augmentation mechanism exists in discs. This endogenous crosslinking may provide mechanical benefits to aging discs, which otherwise deteriorate due to mechanical or chemical degeneration. Notably, a lower level of pentosidine crosslinks was observed in severely degenerated discs [19]. The absence of these age-related crosslinks in severely degenerated discs may signal their importance in maintaining tissue integrity in aging discs. Likewise, disc crosslink augmentation may provide mechanical advantages towards resisting the ongoing process of disc matrix degradation.

Genipin is a gardenia fruit extract that is known to be an effective collagen crosslinker. It is thought to link amino acid groups in intramolecular, intermolecular, and intermicrofibrillar bonds [20]. Genipin crosslinking with the collagenous tissues has demonstrated enzymatic stability [21], subcutaneous biocompatibility [22], and relatively low cytotoxicity [23] and genotoxicity [24]. Genipin crosslinking has been suggested as a possible treatment for resisting disc degeneration and reducing low back pain due to its demonstrated capabilities to improve intervertebral joint stability [25-27], increase AF strength and toughness [28], and double nutritional flow through the disc [29]. Crosslink augmentation of the disc can be administered locally via micro-invasive surgery, considering the structural uniqueness of the disc as an isolated, confined space, where a single injection can be effective [30, 31]. The biocompatibility associated with direct use of genipin in the disc is considered outside of the scope of the present investigation. However, a recent animal study demonstrated that genipin reagent is able to react with a stabinjured disc in vivo (AF, NP, or both) without any obvious morbidity, where the exogenous crosslinking restored the disc integrity following a short-term injection treatment [32].

In a previous study investigating the exogenous crosslinking effects on IDP, it was noted that IDP was lower in genipin-crosslinked disc specimens under axial compressive load and that the drop in IDP caused by creep loading was decreased [33]. In seeming opposition, another work revealed that genipin crosslinking doubled fluid flow into and out of the NP following compressive creep loading and recovery [29]. To harmonize these results, it was postulated that crosslink augmentation causes material property changes that affect compressive load sharing mechanisms in the disc. To characterize the load sharing variations that result from exogenous crosslink augmentation, the present study models and analyzes stress distribution changes using in vitro measurements of disc deformation and the known material property changes [28]. The specific, directional (circumferential tension-CT, radial compression-RC, lamellae-aligned tension-AT, and axial compression-AC) material property changes associated with genipin crosslinking of isolated AF specimens were described in detail in a previous study [28]. In the present study, simultaneous intervertebral disc deformations were measured. Combined with the previously determined anisotropic material properties, these data enabled calculation of tissue stresses and exogenouscrosslinking-induced changes in load sharing. An experimental model under static creep loading was chosen to both 
be consistent with previous studies [29, 33] and provide an indication of load sharing changes at differing levels of disc fluid content.

To our knowledge, no previous studies have measured external radial bulging and internal disc deformations simultaneously. Intradiscal deformations have been measured previously using implanted beads and threads $[34,35]$. Internal disc deformations have also been measured using monofilament nylon threads implanted into the disc in the anteroposterior direction, allowing intradiscal displacement to be traced by magnetic resonance images [36]. Both of these techniques may introduce errors in the form of unknown movement between the implanted materials and annular lamellae. In the present study, two zero-contact methods were developed to quantify internal and external disc deformations in the transverse plane: a sonographic imaging technique directly measured internal deformation of AF, while a custom rotating laser measurement system simultaneously quantified disc bulge. Limited access to the sonographic equipment used in this study (one day) limited the number of total experiments conducted to eight.

\section{Materials and Methods}

A total of eight bovine lumbar motion segments (4- to 6-month-old) were used in this study. Specimen preparation included cutting the pedicles and removing the posterior processes and the soft tissue around the disc. Great care was taken to maintain disc hydration using a saline mister at regular intervals. The specimens were randomly treated by either soaking in phosphate-buffered saline (PBS) solution as a control group $(\mathrm{n}=4)$, or soaking in $0.33 \%(\mathrm{~g} / \mathrm{g})$ genipin $(98 \%$ pure, Challenge Bioproducts Co., Taichung, Taiwan) plus PBS solution as a crosslinked group $(n=4)$. The specimens were soaked for two days at room temperature in their respective solutions before being tested. Soaking was used rather than injections of reagent in order to minimize variation of treatment coverage, which could potentially mask the treatment effects. A previous study verified that soaking treatment does not affect disc tissue hydration [33].

After individual treatment, the superior and inferior vertebrae of the motion segment specimens were potted in polyurethane for mechanical testing. An axial compressive load of $75 \mathrm{~N}$ was applied to the specimens by an Instron 8521 material testing system (Instron Corp., Canton, MA, USA) and held for $10 \mathrm{~min}$ for preconditioning. Continuously, the specimens were loaded by an axial compressive load of $750 \mathrm{~N}$ at a loading rate of $25 \mathrm{~N} / \mathrm{s}$, and then held at a constant load for a 1-h creep period. Disc height was simultaneously recorded by the Instron machine with a data acquisition rate of $10 \mathrm{~Hz}$.
The external surface contour of the mid-transverse plane of the disc and the internal deformation of the $\mathrm{AF}$ were measured at three time points: (1) immediately after the 75-N compressive load was applied, (2) immediately after the 750-N compressive load was applied, and (3) after $1 \mathrm{~h}$ of creep loading but before ramp unloading. The external surface contour of the disc in the transverse plane was measured by a custom non-contact measurement system containing a rotating laser displacement sensor (LK-081, Keyence Corp., Elmwood Park, NJ, USA) (Fig. 1) with a resolution of $3 \mu \mathrm{m}$ and a system accuracy of $0.01 \mathrm{~mm}$. The measurement was started each time at the same disc location, marked by a colored marker at the mid-axial level on the disc surface, where the largest expected bulging would occur. The rate of data acquisition was $20 \mathrm{~Hz}$, with 800 data points for a $360^{\circ}$ complete circle. Circumference length and radius of the disc were calculated using custom-designed software based on the Matlab (MathWorks, Natick, MA, USA) programming environment. The radius data were further used for calculation of the lamellae-aligned tensile strain. Quasi-static assumptions allowed the circumferential tensile strain to be calculated as follows:

$\varepsilon_{\mathrm{CTx}}=\frac{C_{\mathrm{x}}-C_{0}}{C_{0}}$

where $\varepsilon_{C T \mathrm{x}}$ is the circumferential tensile strain, $C_{0}$ is the contour length at a 75-N applied load, $C_{1}$ is the circumferential length measured immediately after application of $750 \mathrm{~N}$, and $C_{2}$ is the circumferential length under load after $1 \mathrm{~h}$ of creep.

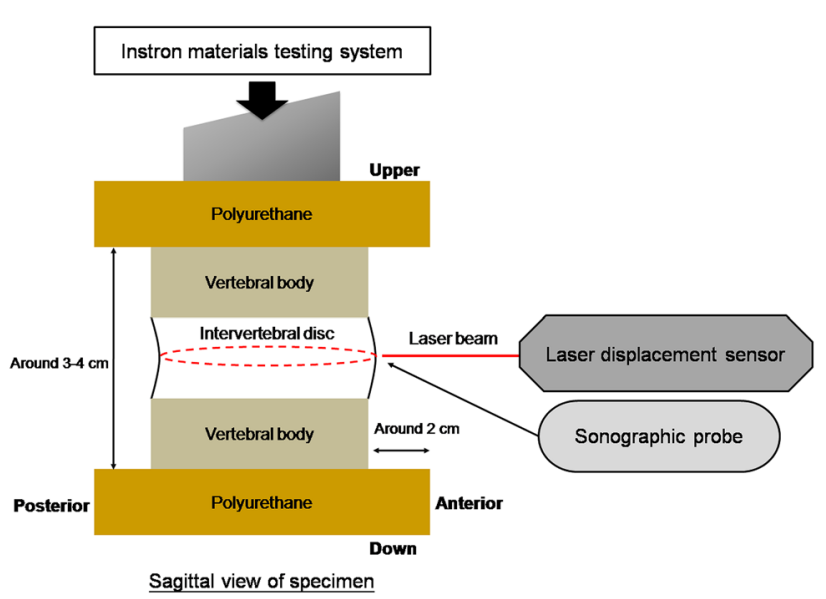

Fig. 1 Test setup. Specimen was loaded using an Instron 8521 system mounted with a non-contact laser measurement system on a platform that rotates around the specimen. The dotted circle near the intervertebral disc represented the track of laser measurement on the circumferential surface of the disc. A 7.5-MHz hand-held sonographic probe was used to collect a simultaneous image of internal deformations 
The internal deformation of disc was measured by an ultrasound imaging machine (280 SL, Johnson \& Johnson Professionals, Inc., Ramsey, NJ, USA) in brightness mode with a 7.5-MHz linear array probe. The probe was applied to the anterior surface of the disc. The width between the outermost and innermost aspect of AF was measured using the built-in "edge (position) reader" of the sonographic machine (Fig. 2). The standard error of the measurements was $0.3 \mathrm{~mm}$. The radial compressive strains were then calculated using the following equation:

$\varepsilon_{\mathrm{RCx}}=\frac{S_{\mathrm{x}}-S_{0}}{S_{0}}$

where $\varepsilon_{R C x}$ is the radial compressive strain, $S_{0}$ is the measured width with a $75-\mathrm{N}$ load, $S_{1}$ is the width at the onset of 750-N loading, and $S_{2}$ is the width after $1 \mathrm{~h}$ of creep but prior to unloading.

The axial compressive strain was calculated based on the disc height data acquired by the Instron machine $( \pm 0.01 \mathrm{~mm})$ using the following equation:

$\varepsilon_{\mathrm{ACx}}=\frac{D H_{\mathrm{x}}-D H_{0}}{D H_{0}}$

where $\varepsilon_{A C \mathrm{x}}$ is the axial compressive strain, $D H_{0}$ is the disc height at a $75-\mathrm{N}$ load, $\mathrm{DH}_{1}$ is the disc height measured immediately after applying $750 \mathrm{~N}$, and $\mathrm{DH}_{2}$ is the disc height after $1 \mathrm{~h}$ of creep.

The lamellae-aligned tensile deformations under compressive load were approximated using circumferential bulging and disc height measurements. The lamellaealigned tensile strain was calculated as the radial expansion minus the bulk expansion:

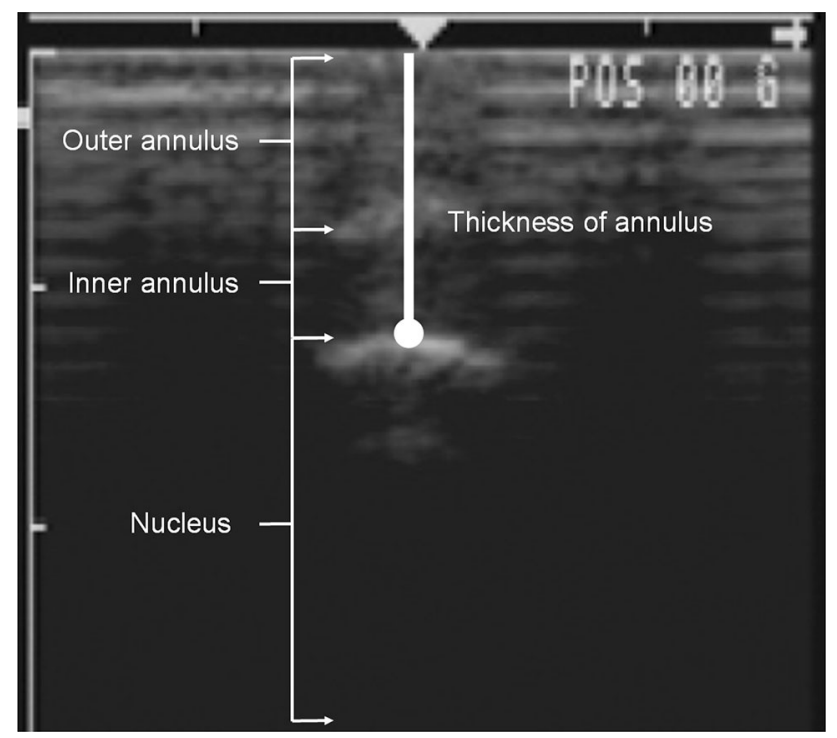

Fig. 2 The thickness of annulus fibrosus was measured in sonographic images at different stages of compressive loading. The left border of the image is marked in centimeters
$\varepsilon_{\mathrm{ATx}}=\frac{\left(R_{\mathrm{x}}-R_{0}\right)-P E_{\mathrm{x}}}{D H_{0}}$

where $\varepsilon_{A T \mathrm{x}}$ is the lamellae-aligned tensile strain, $R_{0}$ is the radius at a $75-\mathrm{N}$ load, $R_{1}$ is the disc radius measured immediately after applying $750 \mathrm{~N}$, and $R_{2}$ is the radius after $1 \mathrm{~h}$ of creep. $P E$ is the bulk expansion in the radial direction based on an equal, assumed Poisson's ratio of 0.4, and the measured axial deformation.

Mean differences between treated and untreated strain measurements were compared using Student's $t$ test. Due to the aforementioned single-day restricted access to the sonographic equipment and an expected level of variability in the data, statistically significant differences were unlikely; consequently, the data trends were also estimated via 95\% confidence intervals (CIs) of the strain differences between treated specimens and controls.

\section{Results}

Radial compressive strain (internal deformation) measurements taken from sonographic images, immediately after $750-\mathrm{N}$ axial loading, were $2.4 \pm 0.8$ and $4.5 \pm 2.2 \%$ (mean and standard deviation, $p=0.123,95 \%$ CI of difference: -4.96 to $0.76 \%$ ) in the control and genipin groups, respectively. After $1 \mathrm{~h}$ of creep loading, the strains were $7.3 \pm 2.4$ and $14.4 \pm 8.2 \%(p=0.148,95 \%$ CI of difference: -17.55 to $3.35 \%$ ) in control and genipin groups, respectively. Axial compressive strains were $13.41 \pm 1.79$ and $11.06 \pm 1.16 \%(p=0.078,95 \%$ CI of difference: -0.35 to $4.95 \%$ ) in control and genipin groups, respectively, when initially loaded to $750 \mathrm{~N}$. After $1 \mathrm{~h}$ of creep loading, the respective strains in control and genipin groups were respectively $36.47 \pm 1.46$ and $32.48 \pm 3.65 \%$ ( $p=0.092,95 \% \mathrm{CI}$ of difference: -0.89 to $8.89 \%$ ). Circumferential strains associated with disc bulge were $2.41 \pm 1.18$ and $0.54 \pm 1.60 \%(p=0.106,95 \% \mathrm{CI}$ of difference: -0.55 to $4.35 \%$ ) in control and genipin groups, respectively, under 750-N load. The circumferential strains increased to $3.90 \pm 2.84$ and $2.60 \pm 0.99 \%(p=0.416$, 95\% CI of difference: -2.34 to $4.94 \%$ ) in control and genipin groups, respectively, after $1 \mathrm{~h}$ of $750-\mathrm{N}$ creep loading. Lamellae-aligned tensile strains, immediately after $750-\mathrm{N}$ axial loading, were calculated to be $8.7 \pm 4.0$ and $1.1 \pm 7.2 \%(p=0.115,95 \% \mathrm{CI}$ of difference: -2.48 to $17.68 \%)$ in the control and genipin groups, respectively. After $1 \mathrm{~h}$ of creep loading, these respective strains increased to $13.4 \pm 13.0$ and $8.5 \pm 3.7 \%(p=0.496,95 \%$ CI of difference: -11.64 to $21.44 \%$ ).

Utilizing modulus data from a previous study [28] and assuming a linear Hooke's relationship between stress and strain, mean tissue stresses were calculated. The results are 
shown in Table 1. Compared to controls, mean axial compressive stresses were approximately doubled in the genipin group for both static and 1-h creep loading of 750-N magnitude (187 and 266\%, respectively). A similar treatmentinduced doubling occurred in the mean radial compressive stresses for both initial loading and after $1 \mathrm{~h}$ of creep loading (200 and 222\%, respectively). Mean axial compressive stresses increased approximately threefold after $1 \mathrm{~h}$ of creep loading compared to stresses at the initial application of a $750-\mathrm{N}$ load for both control and genipin groups (272 and $386 \%$, respectively). A similar threefold increase due to creep loading was seen in the mean radial compressive stresses (300\% for controls, 333\% for genipin crosslinking). Mean tensile stress increases due to creep loading were $64 \%$ for circumferential and $56 \%$ for lamellae-aligned tension in the controls. Much larger mean tensile stress increases due to creep loading were seen in genipin-treated specimens, 385 and $800 \%$ for circumferential and lamellae-aligned tensile stresses, respectively. Yet, at the end of $1 \mathrm{~h}$ of compressive creep loading, the genipin-crosslinked discs experienced similar stress (18\% less in circumferential tension and $28 \%$ less in lamellae-aligned tension) compared with that of controls on average. Under transient loading conditions, mean tensile stresses in the crosslinked discs were 3.5 and 8 times less than those of controls in circumferential and lamellae-aligned tension, respectively.

\section{Discussion}

Simultaneous non-contact radial disc bulge, disc height, and internal disc deformation measurements were made on bovine intervertebral discs subjected to sustained compressive loading of intact motion segments. Combined with the material properties previously measured from isolated AF specimens [28], these data enabled an analysis of load sharing changes brought about by genipin crosslink augmentation.

One observation from these data is the substantial reduction of the tensile strain associated with disc bulging with crosslink augmentation. Although not established beyond the condition of compressive creep loading used in the present study, reduction of disc bulging under load could have direct implications on neural compression and pain in the symptomatic disc. This parameter should be further evaluated under additional pseudo-physiologic loading profiles, including compression with bending. Nonetheless, these strain observations suggest that exogenous crosslinking treatment for the disc could potentially have benefits in regard to pain relief.

In the present study, it was found that average axial and radial compressive stresses of the annulus increased approximately two-fold due to crosslink augmentation at the onset of a 750-N load. Conversely, average lamellaealigned and circumferential tensile stresses were decreased up to eight- and three-fold, respectively, due to exogenous crosslinking. Over $1 \mathrm{~h}$ of creep loading, the two compressive stresses in both control and genipin-crosslinked groups increased at comparable rates based on a percentage of initial stress. The two tensile stresses in the genipincrosslinked group approached but did not reach the respective levels of the control means over the period of creep loading. These results suggest that the type of compressive load support was transformed from resembling a fluid pressurized, axially loaded elastic container to resembling a thick-walled, axially loaded elastic cylinder.

Table 1 Stress distributions in annulus fibrosus of control and genipin groups under compressive creep loading

\begin{tabular}{|c|c|c|c|c|c|c|}
\hline \multirow[t]{2}{*}{ Compressive properties } & \multicolumn{3}{|l|}{$\mathrm{AC}$} & \multicolumn{3}{|l|}{$\mathrm{RC}$} \\
\hline & Initial & Creep & Creep/initial (\%) & Initial & Creep & Creep/initial $(\%)$ \\
\hline Control (MPa) & 0.46 & 1.25 & $272 \%$ & 0.03 & 0.09 & $300 \%$ \\
\hline Genipin (MPa) & 0.86 & 3.32 & $386 \%$ & 0.06 & 0.20 & $333 \%$ \\
\hline Genipin/control (\%) & $187 \%$ & $266 \%$ & & $200 \%$ & $222 \%$ & \\
\hline \multirow[t]{2}{*}{ Tensile properties } & \multicolumn{3}{|l|}{$\mathrm{CT}$} & \multicolumn{3}{|l|}{ AT } \\
\hline & Initial & Creep & Creep/initial (\%) & Initial & Creep & Creep/initial (\%) \\
\hline Control (MPa) & 0.47 & 0.77 & $164 \%$ & 0.16 & 0.25 & $156 \%$ \\
\hline Genipin (MPa) & 0.13 & 0.63 & $485 \%$ & 0.02 & 0.18 & $900 \%$ \\
\hline Genipin/control (\%) & $28 \%$ & $82 \%$ & & $13 \%$ & $72 \%$ & \\
\hline
\end{tabular}

Mean values are given. Stress unit: $\mathrm{MPa}$

$A C$ axial compression, $R C$ radial compression, $C T$ circumferential tension, $A T$ lamellae-aligned tension

Initial stress represents the stress difference generated during the process of ramping from a load of $75 \mathrm{~N}$ to $750 \mathrm{~N}$. Creep stress represents the stress generated from the $75-\mathrm{N}$ load to $1 \mathrm{~h}$ of creep at $750 \mathrm{~N}$. Tensile stresses were decreased in the genipin-crosslinked discs while compressive stresses were increased 
In both static and creep loading conditions, a greater proportion of external compressive load was sustained by compressive stresses in the $\mathrm{AF}$ of the crosslinked discs compared to the control group. A previous study of crosslink augmentation effects on disc material properties demonstrated significant increases in circumferential and lamellae-aligned tensile yield strength, ultimate strength, and toughness [28]. Combined with the observed drop in tensile stress in the present study, these results bode well for the disc's demanding mechanical environment in view of the propensity for tensile overload of posterior annular tissue when subjected to flexion-bending, as described by numerous authors [37-39]; however, the present study did not include bending loads.

This drop in tensile loading and increase in compressive loading in the AF corresponds with previous IDP results, which showed an initially lower IDP with a 750-N compressive creep load in the genipin group as compared to the control group [33]. Likewise, crosslinking reduced the loss of IDP (disc depressurization) during compressive creep loading [33]. In another set of experiments investigating hydration changes in different disc regions under a given compressive creep loading, it was demonstrated that outward fluid flow through the disc doubled in the genipin group as compared to that in the control group [29]. One would expect that greater fluid transport would correspond to accelerated loss of hydrostatic load support rather than the observed maintenance of IDP. The results presented in the present study suggest that the flow-induced drop in IDP may be moderated by the increased proportion of annular compressive load support associated with increased crosslinking.

Intervertebral disc degeneration involves a change of tensile and compressive properties of the AF, which, in turn, could influence the annulus fatigue resistance properties [40-43], and the propensity for pain-related failures of the disc such as bulges or herniations that impinge or compress adjacent neural tissues. Tensile strength and stiffness of the AF decrease with aging and degeneration [40, 44, 45]. Loss of tensile strength may lead to demand on the AF to support an increasing amount of axial compressive stress, which could further deteriorate the material properties of the AF, leading to a vicious cycle of degeneration that could lead to disc collapse and low back pain. Crosslink augmentation increases AF lamellae-aligned and circumferential tensile moduli and strength (yield strength increases of 45 and $78 \%$, respectively) [28], while changing the mechanism of compressive load support according to the findings of the present study. Thus, one could suggest that crosslink augmentation may work towards preventing potential annular failures associated with disc bulges and herniations in moderately degenerated discs by both reducing lamellae-aligned and circumferential tensile stresses while dramatically increasing tensile strength [28].

To our knowledge, the technique used to measure internal disc radial compressive deformations using ultrasound equipment has not been reported previously. The standard deviation of repeated measurements was approximately $0.3 \mathrm{~mm}$, which is more than the mean difference of radial compressive strain between crosslinked and control groups at initial loading and less than the mean difference after creep loading. The determination of the margin between the NP and the AF was made via visual observation by one trained observer, which likely contributed towards the measurement error. Care was taken to place the probe position and angle similarly with successive measurements, but probe position is also a potential source of errors. Various techniques could be employed to minimize measurement errors, including digital edge detection of the margin between NP and inner AF, and the design of an apparatus to hold the sonographic probe and register the position and angle relative to the specimen. Similarly, the lamellae-aligned tensile deformation measurement can be further refined by developing a method to directly measure this deformation. Several methods were attempted such as measuring the changes of the contours of the disc under load from digital images, tracing the lateral bulging pointto-point on the surface with monofilament nylon, and analyzing the contours of press-molds obtained from applying molding material to the disc surface. All of these measurement techniques provided inadequate repeatability or accuracy. Relying instead on the considerably more accurate measurements of circumferential bulging and disc height, lamellae-aligned tensile strain was approximated as radial expansion minus bulk expansion based on measured axial deformation and using an equal, assigned Poisson's ratio of 0.4 .

Caution should be exercised in making inferences about the mechanical effects of age-related increases in crosslinking of intervertebral discs based on the results of this study. Tensile strength and stiffness are known to decrease with aging and degeneration, at the same time that crosslinking generally increases. This may suggest that the opposing effects of endogenous crosslink augmentation are generally not sufficient to compensate for the declining mechanical properties of the matrix. To our knowledge, no data has been published that demonstrates the isolated effects of age-related crosslinks on AF yield strength, ultimate strength, and toughness. It is also interesting that Duance et al. found that the most severely degenerated discs had lower quantities of age-related crosslinks [19]. Future studies should analyze the effects of endogenous, age-related crosslinking on AF material properties and load sharing behavior in order to determine the extent to which 
endogenous crosslinking is beneficial to the mechanical integrity of aging disc tissue.

\section{Conclusion}

This study demonstrated that the exogenous crosslinking could change the compressive load sharing mechanism of intervertebral disc. Under the given static and creep compressive loading, the tensile stresses in the disc were decreased while the compressive stresses were increased correspondingly following genipin treatment. As the tensile properties of AF were known important for maintaining disc integrity during disc bulging outwards, these results have implication that crosslink augmentation may be advantageous for the degraded or degenerated disc which has insufficient mechanical performance.

Open Access This article is distributed under the terms of the Creative Commons Attribution 4.0 International License (http://crea tivecommons.org/licenses/by/4.0/), which permits unrestricted use, distribution, and reproduction in any medium, provided you give appropriate credit to the original author(s) and the source, provide a link to the Creative Commons license, and indicate if changes were made.

\section{References}

1. Adams, M. A., \& Roughley, P. J. (2006). What is intervertebral disc degeneration, and what causes it? Spine (Phila Pa 1976), 31(18), 2151-2161.

2. Adams, M. A., Freeman, B. J., Morrison, H. P., Nelson, I. W., \& Dolan, P. (2000). Mechanical initiation of intervertebral disc degeneration. Spine (Phila Pa 1976), 25(13), 1625-1636.

3. Brown, K. R., Pollintine, P., \& Adams, M. A. (2008). Biomechanical implications of degenerative joint disease in the apophyseal joints of human thoracic and lumbar vertebrae. American Journal of Physical Anthropology, 136(3), 318-326.

4. Panjabi, M. M. (1992). The stabilizing system of the spine. Part I. Function, dysfunction, adaptation, and enhancement. J Spinal Disord, 5(4), 383-389. discussion 397.

5. Ohshima, H., Tsuji, H., Hirano, N., Ishihara, H., Katoh, Y., \& Yamada, H. (1989). Water diffusion pathway, swelling pressure, and biomechanical properties of the intervertebral disc during compression load. Spine (Phila Pa 1976), 14(11), 1234-1244.

6. Ohshima, H., \& Urban, J. P. (1992). The effect of lactate and $\mathrm{pH}$ on proteoglycan and protein synthesis rates in the intervertebral disc. Spine (Phila Pa 1976), 17(9), 1079-1082.

7. Ohshima, H., Urban, J. P., \& Bergel, D. H. (1995). Effect of static load on matrix synthesis rates in the intervertebral disc measured in vitro by a new perfusion technique. Journal of Orthopaedic Research, 13(1), 22-29.

8. Matsumoto, T., Kawakami, M., Kuribayashi, K., Takenaka, T., \& Tamaki, T. (1999). Cyclic mechanical stretch stress increases the growth rate and collagen synthesis of nucleus pulposus cells in vitro. Spine (Phila Pa 1976), 24(4), 315-319.

9. Rannou, F., Poiraudeau, S., Foltz, V., Boiteux, M., Corvol, M., \& Revel, M. (2000). Monolayer anulus fibrosus cell cultures in a mechanically active environment: Local culture condition adaptations and cell phenotype study. Journal of Laboratory and Clinical Medicine, 136(5), 412-421.

10. Rannou, F., Richette, P., Benallaoua, M., Francois, M., Genries, V., Korwin-Zmijowska, C., et al. (2003). Cyclic tensile stretch modulates proteoglycan production by intervertebral disc annulus fibrosus cells through production of nitrite oxide. Journal of Cellular Biochemistry, 90(1), 148-157.

11. Lotz, J. C., Colliou, O. K., Chin, J. R., Duncan, N. A., \& Liebenberg, E. (1998). Compression-induced degeneration of the intervertebral disc: An in vivo mouse model and finite-element study. Spine (Phila Pa 1976), 23(23), 2493-2506.

12. Iatridis, J. C., Mente, P. L., Stokes, I. A., Aronsson, D. D., \& Alini, M. (1999). Compression-induced changes in intervertebral disc properties in a rat tail model. Spine (Phila Pa 1976), 24(10), 996-1002.

13. Lotz, J. C., \& Chin, J. R. (2000). Intervertebral disc cell death is dependent on the magnitude and duration of spinal loading. Spine (Phila Pa 1976), 25(12), 1477-1483.

14. Stokes, I. A., \& Iatridis, J. C. (2004). Mechanical conditions that accelerate intervertebral disc degeneration: overload versus immobilization. Spine (Phila Pa 1976), 29(23), 2724-2732.

15. Nachemson, A. L. (1981). Disc pressure measurements. Spine (Phila Pa 1976), 6(1), 93-97.

16. Burgeson, R. E., \& Nimni, M. E. (1992). Collagen types. Molecular structure and tissue distribution. Clinical Orthopaedics and Related Research, 282, 250-272.

17. Eyre, D. R., Dickson, I. R., \& Van Ness, K. (1988). Collagen cross-linking in human bone and articular cartilage. Age-related changes in the content of mature hydroxypyridinium residues. Biochemical Journal, 252(2), 495-500.

18. Pokharna, H. K., \& Phillips, F. M. (1998). Collagen crosslinks in human lumbar intervertebral disc aging. Spine (Phila Pa 1976), 23(15), 1645-1648.

19. Duance, V. C., Crean, J. K., Sims, T. J., Avery, N., Smith, S., Menage, J., et al. (1998). Changes in collagen cross-linking in degenerative disc disease and scoliosis. Spine (Phila Pa 1976), 23(23), 2545-2551.

20. Sung, H. W., Chang, W. H., Ma, C. Y., \& Lee, M. H. (2003). Crosslinking of biological tissues using genipin and/or carbodiimide. Journal of Biomedical Materials Research, Part A, 64(3), $427-438$.

21. Sung, H. W., Liang, I. L., Chen, C. N., Huang, R. N., \& Liang, H. F. (2001). Stability of a biological tissue fixed with a naturally occurring crosslinking agent (genipin). Journal of Biomedical Materials Research, 55(4), 538-546.

22. Huang, L. L., Sung, H. W., Tsai, C. C., \& Huang, D. M. (1998). Biocompatibility study of a biological tissue fixed with a naturally occurring crosslinking reagent. Journal of Biomedical Materials Research, 42(4), 568-576.

23. Sung, H. W., Huang, R. N., Huang, L. L., \& Tsai, C. C. (1999). In vitro evaluation of cytotoxicity of a naturally occurring crosslinking reagent for biological tissue fixation. Journal of Biomaterials Science-Polymer Edition, 10(1), 63-78.

24. Tsai, C. C., Huang, R. N., Sung, H. W., \& Liang, H. C. (2000). In vitro evaluation of the genotoxicity of a naturally occurring crosslinking agent (genipin) for biologic tissue fixation. Journal of Biomedical Materials Research, 52(1), 58-65.

25. Hedman, T. P., Saito, H., Vo, C., \& Chuang, S. Y. (2006). Exogenous cross-linking increases the stability of spinal motion segments. Spine (Phila Pa 1976), 31(15), E480-485.

26. Yerramalli, C. S., Chou, A. I., Miller, G. J., Nicoll, S. B., Chin, K. R., \& Elliott, D. M. (2007). The effect of nucleus pulposus crosslinking and glycosaminoglycan degradation on disc mechanical function. Biomechanics and Modeling in Mechanobiology, 6(1-2), 13-20. 
27. Barbir, A., Michalek, A. J., Abbott, R. D., \& Iatridis, J. C. (2010). Effects of enzymatic digestion on compressive properties of rat intervertebral discs. Journal of Biomechanics, 43(6), 1067-1073.

28. Chuang, S. Y., Odono, R. M., \& Hedman, T. P. (2007). Effects of exogenous crosslinking on in vitro tensile and compressive moduli of lumbar intervertebral discs. Clinical Biomechanics, 22(1), 14-20.

29. Chuang, S. Y., Popovich, J. M., Jr., Lin, L. C., \& Hedman, T. P. (2010). The effects of exogenous crosslinking on hydration and fluid flow in the intervertebral disc subjected to compressive creep loading and unloading. Spine (Phila Pa 1976), 35(24), E1362-1366.

30. Thompson, J. P., Oegema, T. R., Jr., \& Bradford, D. S. (1991). Stimulation of mature canine intervertebral disc by growth factors. Spine (Phila Pa 1976), 16(3), 253-260.

31. Masuda, K., Oegema, T. R., Jr., \& An, H. S. (2004). Growth factors and treatment of intervertebral disc degeneration. Spine (Phila Pa 1976), 29(23), 2757-2769.

32. Lin, H. J., Lin, L. C., Hedman, T. P., Chen, W. P., \& Chuang, S. Y. (2015). Exogenous crosslinking restores intradiscal pressure of injured porcine intervertebral discs: An in vivo examination using quantitative discomanometry. Spine (Phila Pa 1976), 40(20), $1572-1577$.

33. Chuang, S. Y., Lin, L. C., \& Hedman, T. P. (2010). The influence of exogenous cross-linking and compressive creep loading on intradiscal pressure. Biomechanics and Modeling in Mechanobiology, 9(5), 533-538.

34. Krag, M. H., Seroussi, R. E., Wilder, D. G., \& Pope, M. H. (1987). Internal displacement distribution from in vitro loading of human thoracic and lumbar spinal motion segments: experimental results and theoretical predictions. Spine (Phila Pa 1976), 12(10), 1001-1007.

35. Seroussi, R. E., Krag, M. H., Muller, D. L., \& Pope, M. H. (1989). Internal deformations of intact and denucleated human lumbar discs subjected to compression, flexion, and extension loads. Journal of Orthopaedic Research, 7(1), 122-131.
36. Kusaka, Y., Nakajima, S., Uemura, O., Aoshiba, H., Seo, Y., \& Hirasawa, Y. (2001). Intradiscal solid phase displacement as a determinant of the centripetal fluid shift in the loaded intervertebral disc. Spine (Phila Pa 1976), 26(9), E174-181.

37. Adams, M. A., \& Hutton, W. C. (1982). Prolapsed intervertebral disc. A hyperflexion injury 1981 Volvo Award in Basic Science. Spine (Phila Pa 1976), 7(3), 184-191.

38. Green, T. P., Adams, M. A., \& Dolan, P. (1993). Tensile properties of the annulus fibrosus II. Ultimate tensile strength and fatigue life. European Spine Journal, 2(4), 209-214.

39. Hedman, T. P., \& Fernie, G. R. (1997). Mechanical response of the lumbar spine to seated postural loads. Spine (Phila Pa 1976), 22(7), 734-743.

40. Acaroglu, E. R., Iatridis, J. C., Setton, L. A., Foster, R. J., Mow, V. C., \& Weidenbaum, M. (1995). Degeneration and aging affect the tensile behavior of human lumbar anulus fibrosus. Spine (Phila Pa 1976), 20(24), 2690-2701.

41. Adams, M. A., \& Dolan, P. (1997). Could sudden increases in physical activity cause degeneration of intervertebral discs? Lancet, 350(9079), 734-735.

42. Best, B. A., Guilak, F., Setton, L. A., Zhu, W., Saed-Nejad, F., Ratcliffe, A., et al. (1994). Compressive mechanical properties of the human anulus fibrosus and their relationship to biochemical composition. Spine (Phila Pa 1976), 19(2), 212-221.

43. Yu, C. Y., Tsai, K. H., Hu, W. P., Lin, R. M., Song, H. W., \& Chang, G. L. (2003). Geometric and morphological changes of the intervertebral disc under fatigue testing. Clinical Biomechanics, 18(6), S3-9.

44. Fujita, Y., Duncan, N. A., \& Lotz, J. C. (1997). Radial tensile properties of the lumbar annulus fibrosus are site and degeneration dependent. Journal of Orthopaedic Research, 15(6), 814-819.

45. Galante, J. O. (1967). Tensile properties of the human lumbar annulus fibrosus. Acta Orthopaedica Scandinavica, 100(Suppl), $1-91$. 